\title{
Correction to: Three new feather mite species (Acariformes: Proctophyllodidae, Trouessartiidae) from tyrant flycatchers (Passeriformes: Tyrannidae) in Brazil
}

Fabio Akashi Hernandes (i)

Published online: 2 February 2022

(C) Springer Nature B.V. 2022

Correction to: Syst Parasitol

https://doi.org/10.1007/s11230-021-10018-0

Following the publication of the original article [1], the legend of Table 1 is omitted.
The correct version of legend is given in this correction, and the original article has been corrected.

The original article can be found online at https:// doi.org/10.1007/s11230-021-10018-0.

F. A. Hernandes $(\square)$

Department of Ecology and Zoology, CCB/ECZ, Federal

University of Santa Catarina (UFSC), Trindade,

Florianópolis, Santa Catarina 88040-970, Brazil

e-mail: abakashi@gmail.com 
Table 1 Feather mite taxa reported from tyrant flycatchers (Tyrannidae) and related families in Brazil.

\begin{tabular}{|c|c|c|c|c|c|c|c|}
\hline \multirow[t]{2}{*}{ Host family } & \multicolumn{6}{|c|}{ Mite taxa } & \multirow[t]{2}{*}{ References* } \\
\hline & \multicolumn{2}{|c|}{ Proctophyllodidae } & \multirow{2}{*}{$\begin{array}{l}\text { Trouessartiidae } \\
\text { Trouessartia }\end{array}$} & \multirow{2}{*}{$\begin{array}{l}\text { Psoroptoididae } \\
\text { Mesalgoides }\end{array}$} & \multirow{2}{*}{$\begin{array}{l}\text { Analgidae } \\
\text { Analges }\end{array}$} & \multirow{2}{*}{$\begin{array}{l}\text { Xolalgidae } \\
\text { Analloptes }\end{array}$} & \\
\hline Tyrannidae & Proctophyllodinae & Pterodectinae & & & & & \\
\hline Arundinicola leucocephala & & $\mathrm{x}$ & $\mathrm{x}$ & $\mathrm{x}$ & & & 18 \\
\hline Camptostoma obsoletum & $\mathrm{x}$ & & $\mathrm{x}$ & & & & 16 \\
\hline Capsiemps flaveola & $\mathrm{x}$ & & & & & & 14 \\
\hline Cnemotriccus fuscatus & $\mathrm{x}$ & $\mathrm{x}$ & $\mathrm{x}$ & $\mathrm{x}$ & & $\mathrm{x}$ & 13,16 \\
\hline Elaenia chilensis & $\mathrm{x}$ & $\mathrm{x}$ & $\mathrm{x}$ & & & & $16, \mathbf{P S}$ \\
\hline Elaenia chiriquensis & $\mathrm{x}$ & $\mathrm{x}$ & $\mathrm{x}$ & & & & $3,4,5,8,9,19$ \\
\hline Elaenia cristata & $\mathrm{x}$ & $\mathrm{x}$ & $\mathrm{x}$ & & & & 5,8 \\
\hline Elaenia flavogaster & $\mathrm{x}$ & $\mathrm{x}$ & $\mathrm{x}$ & & & & $2,5,8$ \\
\hline Elaenia spectabilis & & $\mathrm{x}$ & $\mathrm{x}$ & & & & 16 \\
\hline Lathrotriccus euleri & $\mathrm{x}$ & $\mathrm{x}$ & $\mathrm{x}$ & & & & $5,8,9$ \\
\hline Machetornis rixosa & & $\mathrm{x}$ & & & & & PS \\
\hline Megarhynchus pitangua & & & & $\mathrm{x}^{*}$ & & & 1,8 \\
\hline Myiarchus tyrannulus & & $\mathrm{x}$ & & & & & $6,7,8$ \\
\hline Myiophobus fasciatus & & $\mathrm{x}$ & $\mathrm{x}$ & & & & 2 \\
\hline Myiopagis gaimardi & $\mathrm{x}$ & $\mathrm{x}$ & & & & & 9 \\
\hline Myiopagis viridicata & $\mathrm{x}$ & $\mathrm{x}$ & $\mathrm{x}$ & & & & 9,16 \\
\hline Pitangus sulphuratus & $\mathrm{x}$ & $\mathrm{x}$ & $\mathrm{x}$ & & & & $4,6,7,8, \mathbf{P S}$ \\
\hline Tyrannus melancholicus & $\mathrm{x}$ & $\mathrm{x}$ & $\mathrm{x}$ & & & & $2,8,17$ \\
\hline Tyrannus savana & & & $\mathrm{x}$ & & & & 12 \\
\hline \multicolumn{8}{|l|}{ Onychorhynchidae } \\
\hline Myiobius atricaudus & $\mathrm{x}$ & & $\mathrm{x}$ & & & & 9,15 \\
\hline \multicolumn{8}{|l|}{ Rhynchocyclidae } \\
\hline Hemitriccus mirandae & & & $\mathrm{x}$ & & & & 2 \\
\hline Hemitriccus striaticollis & & $\mathrm{x}$ & & & & & 10,16 \\
\hline Hemitriccus margaritaceiventer & & $\mathrm{x}$ & $\mathrm{x}$ & & & & 10,16 \\
\hline Hemitriccus furcatus & & $\mathrm{x}$ & & & & & 10 \\
\hline Mionectes rufiventris & $\mathrm{x}$ & $\mathrm{x}$ & & & & & 5,8 \\
\hline Phylloscartes kronei & $\mathrm{x}$ & & $\mathrm{x}$ & & & & 11,12 \\
\hline Leptopogon amaurocephalus & $\mathrm{x}$ & & $\mathrm{x}$ & & $\mathrm{x}$ & & 9,14 \\
\hline Corythopis delalandi & $\mathrm{x}$ & & & & $\mathrm{x}$ & & $4,5,8,9$ \\
\hline Tolmomyias flaviventris & $\mathrm{x}$ & & & & & & 9 \\
\hline Tolmomyias sulphurescens & $\mathrm{x}$ & & $\mathrm{x}$ & & & & $5,8,9$ \\
\hline
\end{tabular}

$* 1=$ Bonnet 1924; 2 = Roda \& Farias 1999; $3=$ Hernandes \& Valim 2006; 4 = Hernandes et al. 2007; $5=$ Kanegae et al. 2008; $6=$ Mironov et al. 2008; $7=$ Valim \& Hernandes 2010; $8=$ Valim et al.2011; $9=$ Enout et al. 2012; $10=$ Hernandes $2013 ; 11=$ Hernandes 2014a; 12 = Hernandes 2014b; 13 = Mironov \& Hernandes 2014; 14 = Daud et al. 2015; $15=$ Hernandes \& Valim 2015; $16=$ Silvaet al. 2015; 17 = Hernandes 2017; 18 = Hernandes \& Flechtmann 2020; $19=$ Valim \& Hernandes 2009; PS = present study.

Publisher's Note Springer Nature remains neutral with regard to jurisdictional claims in published maps and institutional affiliations. 